\title{
Synthesis and Antimicrobial activity of some new Pyrimidines of 6-chlorobenzimidazoles
}

\author{
INDIRA M. MADAWALI, NAVANATH V. KALYANE, GAVIRAJ E. N. and SHIVAKUMAR B* \\ Department of Pharmaceutical Chemistry, B. L. D. E. A's SSM College of Pharmacy \\ and Research Centre, Vijayapur, Karnataka-586 103, India. \\ *Corresponding author E-mail: drbsk_2007@yahoo.com \\ http://dx.doi.org/10.13005/ojc/340358
}

(Received: April 02, 2018; Accepted: June 05, 2018)

\section{ABSTRACT}

\begin{abstract}
A new series of pyrimidines of 6-chlorobenzimidazoles have been synthesized by the reaction of chalcone derivatives of 6-chlorobenzimidazole with guanidine nitrate in ethanol and aqueous solution of sodium hydroxide for evaluating them as potent antimicrobial agents. Results reveal that, compounds exhibited significant antibacterial and antifungal activities.
\end{abstract}

Keywords: Benzimidazoles, Chalcones, Pyrimidines, Antimicrobial activity.

\section{INTRODUCTION}

Benzimidazole nucleus is one of the bioactive heterocyclic compounds exhibiting a range of biological activities ${ }^{1}$. Due to a wide range of pharmacological activities of benzimidazoles, synthesis has been found a significant attention now a day.

To, develop novel quinoline based fused heterocyclic systems; a quinoline nucleus with different substituent's was required, which afforded a versatile synthetic procedure for further heterocycles ${ }^{2}$.

Synthesis of pyrimidines has been advanced and developing field in the heterocyclic chemistry.
Pyrimidines have the crucial value of their biological and pharmacological properties. $\alpha, \beta$-unsaturated ketones are reported to react with guanidine to give the unstable dihydropyrimidines which get dehydrogenated to form 2-aminopyrimidines ${ }^{3}$.

Pyrimidine ring complexes with various heterocyclic molecules and found to be an essential part of the natural products, agrochemicals and veterinary medical products. Fused pyrimidine derivatives have attracted many researchers over many years, due to their important biological activities ${ }^{4}$. The preclinical data from the literature survey reveals that the heterocycles in conjunction with the pyrimidine have shown good antimicrobial $^{5}$, antioxidant ${ }^{6}$, anti-inflammatory ${ }^{7}$, analgesic and antipyretic ${ }^{8}$, anti-tumor activities ${ }^{9}$. In

This is an Open Access article licensed under a Creative Commons Attribution-Non Commercial-Share Alike 4.0 International License (https://creativecommons.org/licenses/by-nc-sa/4.0/), which permits unrestricted Non Commercial use, distribution and reproduction in any medium, provided the original work is properly cited. 
particular,bezimidazolo-pyrimidine derivatives were found as strong antimicrobial agents ${ }^{10,11}$.

Antimicrobial agents are one of the most important weapons in the resistance of infection caused by bacterial strains. In the last few years, increase the resistance of micro-organisms toward antimicrobial agents is a serious health problem, so there is a need for safe, effective and novel antimicrobial agents ${ }^{12}$.

By seeing impressive biological profile of benzimidazoles, quinolines and pyrimidines and also with respect to our work in synthesis and evaluation of biologically active new heterocycles, we planned to synthesize the new series of pyrimidines of 6 -chlorobenzimidazoles as potential antimicrobial agents.

\section{MATERIALS AND METHODS}

All the solvents and reagents were bought and used as such. The fusion points are examined by open capillary tube method and are uncorrected. By the use of $\mathrm{KBr}$ pellet technology IR spectra of compounds on Shimadzu FTIR spectrometer 8400 $S$ were noted. NMR spectra were seized on Bruker Avance II of 400 NMR spectrometer.

\section{Synthesis of 6-chloro-2-(a-hydroxyethyl)benzimidazole(II)}

An equimolar amount of 4-chloro-Ophenylenediamine (0.01 mole) and lactic acid $(0.01$ mole), $4 \mathrm{~N} \mathrm{HCl}$ are refluxed in synthetic microwave oven with the intensity of $65 \%$ (450 W) for a period of 190 minutes. The conclusion of the reaction was supervised by TLC, and then the reaction mixture was neutralized with sodium bicarbonate. The filtered product was washed and dried with water. Further, it was purified by recrystallization from ethanol. m. p. $194-95^{\circ} \mathrm{C}^{13,14,15}$.

\section{Synthesis of 6-chloro-2-acetylbenzimidazole(III)}

To a reaction mixture of 6 -chloro-2- $(\alpha$ hydroxyethyl)benzimidazole $(9.8 \mathrm{~g}, 50 \mathrm{mmol})$ in Dilute $\mathrm{H}_{2} \mathrm{SO}_{4}(5 \% ; 40 \mathrm{ml})$ with stirring, a solution of $\mathrm{K}_{2} \mathrm{Cr}_{2} \mathrm{O}_{7}(44 \mathrm{~g}, 150 \mathrm{mmol})$ in aqueous $\mathrm{H}_{2} \mathrm{SO}_{4}$ (25\%, V/V; $80 \mathrm{ml}$ ) was taken up during a period of 20 minutes drop wise.Continuing the agitation for $2 \mathrm{~h}$, at an ambient temperature, the solid (which is the chromium complex) separates out, was suspended in $50 \mathrm{ml}$ of water. The $\mathrm{pH}$ up to 6-6.5 was adjusted with aqueous ammonia (1:1). Then the solid product filtered, washed with water and dried. The product further recrystallized from ethyl acetate. m. p. $198-99^{\circ} \mathrm{C}^{16}$.

\section{General procedure for the synthesis of chalcone} derivative of 6-chlorobenzimidazoles (IVa)

To a reaction mixture of 6 -chloro-2acetylbenzimidazole $(10 \mathrm{mmol}, 1.94 \mathrm{~g})$ in aqueous $\mathrm{NaOH}(10 \%, 30 \mathrm{ml})$, respective 2-chloroquinoline3-carbaldehyde (10 mmol, $1.91 \mathrm{~g}$ ) were added and agitated for $30 \mathrm{~min}$. at an ambient temperature. After the conclusion of the reaction the solid product was filtered, washed and dried with water. Further, it was recrystallized from ethanol ${ }^{17,18,19}$.

\section{Chalcone derivatives of 6-chlorobenzimidazoles (IVa-k) \\ IVa: Yellow solid, yield 76\%, m.p. 252-} $254^{\circ} \mathrm{C}$; IR (KBr): 3300, 3050, 2800, 1700, 1600, 1522, 1475,1250, 875, 800,750 $\mathrm{cm}^{-1} ;{ }^{1} \mathrm{H} \mathrm{NMR}\left(\mathrm{CDCl}_{3}\right.$, $400 \mathrm{MHz}$ ): $\delta$ 7.69-7.71 (d, $1 \mathrm{H}, \mathrm{CO}-\mathrm{CH}=\mathrm{CH}), 7.71-$ 7.69 (d, J=8Hz), 7.27-8.29 (m, 8H, ArH), 8.01-7.99 (d, J=8Hz), 8.65 (s, 1H, NH).

IVb: Yellow solid, yield 68\%, m.p. 242$244^{\circ} \mathrm{C}$; IR (KBr): 3350, 3100, 2800, 1700, 1600, 1550, 1475, 1350, 775, $700 \mathrm{~cm}^{-1} ;{ }^{1} \mathrm{H} \mathrm{NMR}\left(\mathrm{CDCl}_{3}\right.$, $400 \mathrm{MHz}): \delta 2.76\left(\mathrm{~s}, 3 \mathrm{H}, \mathrm{CH}_{3}\right), 7.48-7.50(\mathrm{~d}, 1 \mathrm{H}, \mathrm{CO}-$ $\mathrm{CH}=\mathrm{CH}), 7.50-7.48(\mathrm{~d}, \mathrm{~J}=8 \mathrm{~Hz}), 7.27-7.78(\mathrm{~m}, 8 \mathrm{H}$, ArH), 7.70-7.68 (d, J=8Hz), $8.65(\mathrm{~s}, 1 \mathrm{H}, \mathrm{NH})$.

IVc: Yellow solid, yield 72\%, m.p. 248$250^{\circ} \mathrm{C}$; IR (KBr): 3300, 3050, 2800, 1700, 1600, 1500, 1450, 1250, 775, $700 \mathrm{~cm}^{-1} ;{ }^{1} \mathrm{H} \mathrm{NMR}\left(\mathrm{CDCl}_{3}\right.$, $400 \mathrm{MHz}): \delta 3.91\left(\mathrm{~s}, 3 \mathrm{H}, \mathrm{OCH}_{3}\right), 7.482-7.487$ (d, $1 \mathrm{H}, \mathrm{CO}-\mathrm{CH}=\mathrm{CH}), 7.487-7.481(\mathrm{~d}, \mathrm{~J}=2.4 \mathrm{~Hz})$, 7.16-7.94 (m, 8H, ArH), 7.94-7.91 (d, J=12Hz), 8.59 (s, $1 \mathrm{H}, \mathrm{NH})$

\section{General procedure for the synthesis of pyrimidines of 6-chlorobenzimidazoles(Va)}

To a refluxing mixture of chalcone $(3.68 \mathrm{~g}$, $0.01 \mathrm{~mol})$ and guanidine nitrate $(1.80 \mathrm{~g}, 0.01 \mathrm{~mol})$ in ethanol (25 ml) was added an aqueous solution of sodium hydroxide $(40 \%, 5 \mathrm{ml})$ portion wise during a period of 3 hours. Refluxing was continued further for 7 hours. The solvent was made to half of its volume. And on cooling, the solid product was separated out. Further, it was filtered, washed with cold aqueous ethanol followed by water and dried. Pure product was obtained by recrystallization from absolute ethanol. 


\section{Pyrimidine derivatives of 6-chlorobenzimidazoles (Va-k)}

Va: Yellow solid, yield $60 \%$, m.p. $83-85^{\circ} \mathrm{C}$; IR (KBr): 3335, 3194, 1602, 1543, 1442, 1253, 825, $715 \mathrm{~cm}^{-1} ;{ }^{1} \mathrm{H} \mathrm{NMR}\left(\mathrm{CDCl}_{3}, 400 \mathrm{MHz}\right): \delta 4.72(\mathrm{~s}, 2 \mathrm{H}$, $\left.\mathrm{NCH}_{2}\right), 7.26-7.74(\mathrm{~d}, \mathrm{~J}=12 \mathrm{~Hz}), 7.26-7.74(\mathrm{~m}, 8 \mathrm{H}$, Ar-H), 7.72-7.70 (d, J=8Hz), 7.84 (s, 1H, NH).

Vb: Yellow solid, yield $48 \%$, m.p. $80-82^{\circ} \mathrm{C}$; IR (KBr): 3327, 3196, 1620, 1543, 1425, 1246, 763, $705 \mathrm{~cm}^{-1} ;{ }^{1} \mathrm{H} \mathrm{NMR}\left(\mathrm{CDCl}_{3}, 400 \mathrm{MHz}\right): \delta 2.64(\mathrm{~s}, 3 \mathrm{H}$, $\mathrm{CH} 3$ ), 4.73 (s, 2H, $\mathrm{NCH}_{2}$ ), 7.19-7.21 (d, J=8Hz), 7.19-7.49 (m, 8H, Ar-H), 7.49-7.47 (d, J=8Hz), 7.88 (s, $1 \mathrm{H}, \mathrm{NH})$.

Vh: Yellow solid, yield 57\%, m.p. $87-89^{\circ} \mathrm{C}$; IR (KBr): 3331, 3196, 1622, 1545, 1442,1222, 812, $775 \mathrm{~cm}^{-1} ;{ }^{1} \mathrm{H} \mathrm{NMR}\left(\mathrm{CDCl}_{3}, 400 \mathrm{MHz}\right): \delta 3.95$ (s, 3H, $\left.\mathrm{OCH}_{3}\right), 4.72\left(\mathrm{~s}, 2 \mathrm{H}, \mathrm{NCH}_{2}\right), 6.94-6.92(\mathrm{~d}, \mathrm{~J}=8 \mathrm{~Hz})$, 6.88-7.68 (m, 8H, Ar-H), 7.54-7.52 (d, J=8Hz), 7.86 (s, $1 \mathrm{H}, \mathrm{NH})$.

Vi: Yellow solid, yield 52\%, m.p. $82-84^{\circ} \mathrm{C}$; IR (KBr): 3198, 3055, 1616, 1577, 1425, 1336, 1246, $763 \mathrm{~cm}^{-1} ;{ }^{1} \mathrm{H} \mathrm{NMR}\left(\mathrm{CDCl}_{3}, 400 \mathrm{MHz}\right): \delta 2.65$ (s, 3H, $\mathrm{CH} 3), 4.75$ (s, $2 \mathrm{H}, \mathrm{NCH}_{2}$ ), 7.22-7.20 (d, J=8Hz), 7.19-7.55 (m, 8H, Ar-H), 7.50-7.48 (d, J=8Hz), 7.90 (s, $1 \mathrm{H}, \mathrm{NH})$.

\section{RESULTS AND DISCUSSION}

Scheme summarizes the synthetic route to obtain 6-chloro-2-( $\alpha$-hydroxyethyl) benzimidazole (II)by reacting 4-chloro-O-phenylenediamine(I) with lactic acid. This on oxidation gives 6-chloro-2acetylbenzimidazole (III). Further condensation with different aromatic/heteroaromatic aldehydes gives chalcone derivatives of 6 -chlorobenzimidazoles (IVa-k). These react with guanidine nitrate in ethanol and aqueous solution of sodium hydroxide gives the title products (Va-k).

The IR spectrum of IVb showed characteristic absorption peaks at $3350(\mathrm{NH}), 1600$ $(\mathrm{C}=\mathrm{O})$ and $1550 \mathrm{~cm}^{-1}(\mathrm{C}=\mathrm{N})$. The ${ }^{1} \mathrm{H}$ NMR spectrum of $\mathrm{IVb}$ displayed a singlet was noticed at $\delta 2.76$, which was assigned to the $\mathrm{CH}_{3}$ protons. A multiplet between $\delta$ 7.27-7.78 $(8 \mathrm{H})$ accounted for aromatic protons. One doublet was observed at $\delta$ 7.48-7.50 accounted for $(\mathrm{CO}-\mathrm{CH}=\mathrm{CH})$ of chalcone moiety.
The IR spectrum of $\mathrm{Vb}$ showed characteristic absorption peaks at $3327(\mathrm{NH}), 1620(\mathrm{C}=\mathrm{O})$ and $1543 \mathrm{~cm}^{-1}(\mathrm{C}=\mathrm{N})$. The ${ }^{1} \mathrm{H}$ NMR spectrum of $\mathrm{Vb}$ displayed a singlet for $\mathrm{CH}_{3}$ proton was observed at $\delta$ 2.64. A singlet of $\mathrm{NCH}_{2}$ protons of pyrimidine nucleus was observed at $\delta$ 4.73. A multiplet of aromatic protons was obtained from 7.19-7.49 and one $\mathrm{NH}$ at 7.88 .

Similarly, the structures of the remaining compounds were confirmed by the spectral data. Further, they have been tested for antimicrobial activity.

\section{Reagents and conditions}

(i) lactic acid, $4 \mathrm{~N} \mathrm{HCl}, \mathrm{MW}$ irradiation 190 min. (ii) $\mathrm{K}_{2} \mathrm{Cr}_{2} \mathrm{O}_{7}, \mathrm{H}_{2} \mathrm{SO}_{4}(25 \% \mathrm{v} / \mathrm{v}) 2 \mathrm{~h}$, (iii) $10 \%$ $\mathrm{NaOH}, 2$-chloroquinoline-3-carbaldehydes, ethanol, 0.5 h, (iv) Guanidine nitrate, ethanol, $\mathrm{NaOH}(40 \%)$, for 10 hours.

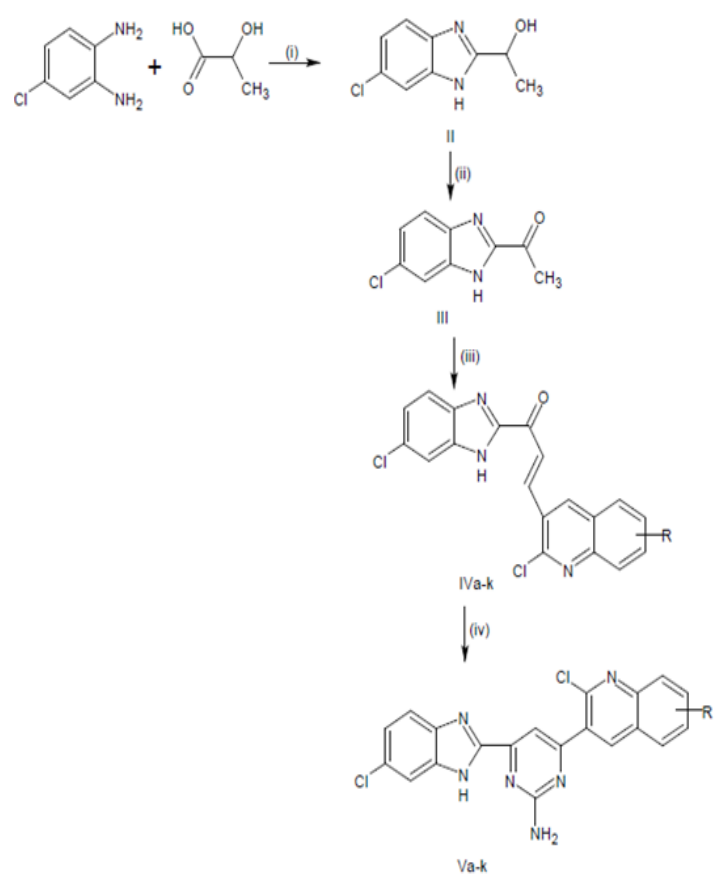

Scheme. $\mathrm{R}=(\mathrm{a})-6-\mathrm{Cl} ;$ (b)-6- $\mathrm{CH}_{3} ;$ (c) $-6-\mathrm{OCH}_{3} ;$ (d)-6-Br; (e)-6-F; (f)-7-Cl; (g)-7- $\mathrm{CH}_{3}$; (h)-7-- $\mathrm{CCH}_{3}$; (i)-8- $\mathrm{CH}_{3}$; (j)-8- $\mathrm{OCH}_{3}$; (k)-H.

\section{Biological Evaluation ${ }^{20-25}$ \\ Antibacterial activity}

The synthesized products have been tested for antibacterial activity against two Grampositive bacteria viz., Bacillus subtilis, Staphylococcus aureus, and two Gram-negative bacteria viz., Proteus Mirabilis and Escheria coli. The standard 
Table 1: Physical Characterization of synthesized compounds (Va-k)

\begin{tabular}{cccccccccc}
\hline SI.No & $\begin{array}{c}\text { Compoud } \\
\text { Code }\end{array}$ & $\mathrm{R}$ & $\begin{array}{c}\text { Molecular } \\
\text { Formula }\end{array}$ & m.p. ${ }^{\circ} \mathrm{C}$ & $\begin{array}{c}\text { Molecular } \\
\text { Weight }\end{array}$ & $\mathrm{N} \%$ & $\mathrm{H} \%$ & $\mathrm{C} \%$ & $\mathrm{Cl} \%$ \\
\hline 1 & $\mathrm{Va}$ & $6-\mathrm{Cl}$ & $\mathrm{C}_{20} \mathrm{H}_{11} \mathrm{Cl}_{3} \mathrm{~N}_{6}$ & 84 & 441.70 & 19.03 & 2.51 & 54.38 & 24.08 \\
2 & $\mathrm{Vb}$ & $6-\mathrm{CH}_{3}$ & $\mathrm{C}_{21} \mathrm{H}_{14} \mathrm{Cl}_{2} \mathrm{~N}_{6}$ & 87 & 421.28 & 19.95 & 3.35 & 59.87 & 16.83 \\
3 & $\mathrm{Vc}$ & $6-\mathrm{OCH}_{3}$ & $\mathrm{C}_{21} \mathrm{H}_{14} \mathrm{Cl}_{2} \mathrm{~N}_{6} \mathrm{O}$ & 87 & 437.28 & 19.22 & 3.23 & 57.68 & 16.22 \\
4 & $\mathrm{Vd}$ & $6-\mathrm{Br}$ & $\mathrm{C}_{20} \mathrm{H}_{11} \mathrm{BrCl}_{2} \mathrm{~N}_{6}$ & 92 & 486.15 & 17.29 & 2.28 & 49.41 & 14.59 \\
5 & $\mathrm{Ve}$ & $6-\mathrm{F}$ & $\mathrm{C}_{20} \mathrm{H}_{11} \mathrm{FCl}_{2} \mathrm{~N}_{6}$ & 94 & 425.25 & 19.76 & 2.61 & 56.49 & 16.67 \\
6 & $\mathrm{Vf}$ & $7-\mathrm{Cl}$ & $\mathrm{C}_{20} \mathrm{H}_{11} \mathrm{Cl}_{3} \mathrm{~N}_{6}$ & 85 & 441.70 & 19.03 & 2.51 & 54.38 & 24.08 \\
7 & $\mathrm{Vg}$ & $7-\mathrm{CH}_{3}$ & $\mathrm{C}_{21} \mathrm{H}_{14} \mathrm{Cl}_{2} \mathrm{~N}_{6}$ & 86 & 421.28 & 19.95 & 3.35 & 59.87 & 16.83 \\
8 & $\mathrm{Vh}$ & $7-\mathrm{OCH}_{3}$ & $\mathrm{C}_{21} \mathrm{H}_{14} \mathrm{Cl}_{2} \mathrm{~N}_{6} \mathrm{O}$ & 89 & 437.28 & 19.22 & 3.23 & 57.68 & 16.22 \\
9 & $\mathrm{Vi}$ & $8-\mathrm{CH}_{3}$ & $\mathrm{C}_{21} \mathrm{H}_{14} \mathrm{Cl}_{2} \mathrm{~N}_{6}$ & 88 & 421.28 & 19.95 & 3.35 & 59.87 & 16.83 \\
10 & $\mathrm{Vj}$ & $8-\mathrm{OCH}_{3}$ & $\mathrm{C}_{21} \mathrm{H}_{14} \mathrm{Cl}_{2} \mathrm{~N}_{6} \mathrm{O}$ & 85 & 437.28 & 19.22 & 3.23 & 57.68 & 16.22 \\
11 & $\mathrm{Vk}$ & $\mathrm{H}$ & $\mathrm{C}_{20} \mathrm{H}_{12} \mathrm{Cl}_{2} \mathrm{~N}_{6}$ & 85 & 407.25 & 20.64 & 2.97 & 58.98 & 17.41 \\
\hline
\end{tabular}

drug used was Ciprofloxacin and DMSO as a solvent. Test products and the standard drug were used at a concentration $100 \mu \mathrm{g} / \mathrm{ml}$ and $50 \mu \mathrm{g} / \mathrm{ml}$. The zones of inhibition of compounds were recorded after incubation of $24 \mathrm{~h}$, at $37^{\circ} \mathrm{C}$.

The results of the antibacterial activity reveals that, the products $\mathrm{Va}, \mathrm{Vb}, \mathrm{Vd}$ and $\mathrm{Vf}$ displayed relatively high antibacterial activity, while products $\mathrm{Ve}, \mathrm{Vg}, \mathrm{Vh}$ and $\mathrm{Vi}$ showed reasonable antibacterial activity. The remaining products showed low activity.

\section{Antifungal activity}

The antifungal activity of the products has been screened against two fungi viz., Aspergilus Niger and Candida albicans by cup-plate method. The standard drug used was Fluconazole and DMSO as a solvent. Test products and standard drug were used at a concentration of $100 \mu \mathrm{g} / \mathrm{ml}$ and $50 \mu \mathrm{g} / \mathrm{ml}$. The zones of inhibition of compounds were recorded after incubation of $48 \mathrm{~h}$, at $25^{\circ} \mathrm{C}$.

Table 2: Antibacterial and antifungal activity of synthesized compounds (Va-k)

\begin{tabular}{ccccccc}
\hline \multirow{2}{*}{ Compound } & \multicolumn{3}{c}{ Antibacterial activitya,b } & \multicolumn{2}{c}{ Antifungal activiy ${ }^{\mathrm{a}, \mathrm{b}}$} \\
& B. subtilis & S. aureus & P. mirabilis & E. coli & A. niger & C. albicans \\
\hline Va & $25 / 21$ & $24 / 20$ & $22 / 21$ & $23 / 20$ & $17 / 15$ & $19 / 16$ \\
Vb & $23 / 21$ & $25 / 24$ & $22 / 20$ & $24 / 23$ & $17 / 15$ & $18 / 16$ \\
Vc & $09 / 06$ & $10 / 08$ & $06 / 05$ & $07 / 05$ & $08 / 05$ & $06 / 04$ \\
Vd & $24 / 22$ & $23 / 20$ & $22 / 20$ & $25 / 23$ & $17 / 15$ & $18 / 15$ \\
Ve & $18 / 16$ & $17 / 14$ & $14 / 13$ & $15 / 14$ & $13 / 11$ & $14 / 12$ \\
Vf & $23 / 21$ & $22 / 19$ & $24 / 22$ & $22 / 20$ & $18 / 16$ & $17 / 15$ \\
Vg & $18 / 15$ & $19 / 16$ & $17 / 15$ & $18 / 16$ & $12 / 11$ & $14 / 12$ \\
Vh & $18 / 16$ & $17 / 15$ & $16 / 14$ & $19 / 17$ & $13 / 10$ & $12 / 11$ \\
Vi & $17 / 16$ & $17 / 15$ & $19 / 17$ & $18 / 16$ & $14 / 13$ & $13 / 11$ \\
Vj & $07 / 05$ & $08 / 05$ & $10 / 07$ & $09 / 06$ & $06 / 03$ & $07 / 04$ \\
Vk & $07 / 05$ & $09 / 07$ & $06 / 05$ & $08 / 06$ & $07 / 05$ & $06 / 03$ \\
Ciprofloxacin $^{\mathrm{b}}$ & 26 & 26 & 26 & 26 & - & - \\
Fluconazole $^{\mathrm{b}}$ & - & - & - & - & 23 & 23 \\
\hline
\end{tabular}

aZone of inhibition at $100 \mu \mathrm{g} / \mathrm{ml}$.

${ }^{\mathrm{b}}$ Zone of inhibition at $50 \mu \mathrm{g} / \mathrm{ml}$.

Minimum inhibitory concentration was found at $40 \mu \mathrm{g} / \mathrm{ml}$ concentration. 
Further from antifungal activity results, products $\mathrm{Va}, \mathrm{Vb}, \mathrm{Vd}$ and $\mathrm{Vf}$ showed excellent results for antifungal activity, while the products $\mathrm{Ve}, \mathrm{Vg}$, Vh and Vi also showed high antifungal activity. The remaining products exhibited low activity.

\section{CONCLUSION}

A simple protocol for the synthesis of some new pyrimidines of 6-chlorobenzimidazoles was developed under synthetic microwave irradiation method and also by the conventional method. It was noted that the preferred synthetic route does an excellent yield and shortened reaction time for benzimidazole synthesis. The synthesized compounds were screened for their antibacterial and antifungal activities. Many of these products showed significant activity.

\section{ACKNOWLEDGEMENT}

The authors are grateful to Vision Group on Science and Technology (VGST), Government of Karnataka state for financial support.

\section{REFERENCES}

1. Salahuddin.;Shaharyar, M.; Mazumdar, A. Arab. J. of chem., 2017, 10(1), 157-173.

2. SinghR. M.; Srivastava, A. Ind. J. of Chem., 2005, 44(B), 1868-1875.

3. Sawhney,S.N.; Vir, D.; Gupta, A. Ind. J. of Chem.,1990, 29(B), 1107-1112.

4. Bhalgat, C. M.; Ramesh, B. Bulletin of Fac. of Pharm. Cairo University., 2014, 52, 259-267.

5. Swaminathan, S.; Ingarsal, N. Orient. J. Chem., 2018, 34 (2), 777-782.

6. Adhikari, A.; Kalluraya, B.; Sujith, K.V.; Gouthamchandra.; Mahmood, R.; Saud. Pharm. J., 2012, 20, 75-79.

7. Bahashwan, S. A.; Fayed, A. A.; Abd El-Galil, E. A.; Eman, M. F.; Kalmouch, A. Molecules., 2013, 18, 15051-15063.

8. Antre, R. V.; Cendilkumar, A.; Goli, D.; Andhale, G. S.; Oswal, R. J. Saud. Pharma. J., 2011, 19, 233-243.

9. Al-Issa, S. A. Saud Pharm. J., 2013, 21, 305-31.

10. Shaaban, M. R.; Saleh, T. S.; Farag, A. M. Heterocycl., 2007, 71(8),1765-1777.

11. Abdelhamid, A. O.; Eman, K. A.; Nadia A. A.; Riheem, A.; Ahmed, S. A. Phosph. Sulf. And Silicon and the Relat. Element., 2010, 185(4), 709-718.

12. Narwal, S.; Kumar S.; Verma, P. K.; Chem. Cent. J., 2017, 11(52), 1-9.

13. Wang, Z. Compreh. Org. Name React. And Reag., 2010, 496, 2197-2199.

14. Reddy, V. M.; Reddy, K. R. Chine. Chem. Lett., 2010, 21, 1145-1148.
15. Mamedov, I. G.; Mamedov, Y. V.; Khrustalev, V. N.; Bayramov, M. R.; Maharramov, A. M. Ind. J. of Chem., 2017, 56 (B), 192-196.

16. Kachroo, M.; Panda, R.;Yadav, R. Der. Pharm. Chemic., 2014, 6(2), 352-359.

17. Sharmila, A. G.; Shivakumar, B.; Gaviraj, E. N. Der. Pharm. Chemic., 2016, 8(5), 33-37.

18. Tabassum, S.; Suresha Kumara, T. H.; Jasinski, J.P.; Millikan, S. N.; Yathirajan, H. S.; SujanGanapathy, P.S.; Sowmya, H. B. V.; More, S. S.; Nagendrappa, G.; Kaur, M.; Jose. G. J. of Molecul. Struct., 2014, 1070, 10-20.

19. Rajakumar, P.; Raja, R. Tetrahed. Lett., 2010, 51, 4365-4370.

20. Vaidehi, B. N. B.; GnanaDeepika, K.; Satya, R. V.; Bangaramma, R. R.; Harish Kumar, R.; RatnaSudha, Y.; Ravi Kumar, T. Inter. J. of Res. in Pharm. And Chem., 2012, 2(2), 322-326.

21. Venkatesan, P.; Sumathi, S. J. of Heterocycl. Chem., 2010, 47, 81-84.

22. Ramanpreet, W.; Hedaitullah, M. D.; Syeda, F. N.; Khalid I.; Lambar, H.S. Inter. J of Res. in Pharm. And Chem., 2011, 1(3), 565-574.

23. Radha, Y .;Manjula, A.;Madhav Reddy, B.; VitthalRao, B. Ind. J. of Chem., 2011, 50(B), 1762-1773.

24. Kalirajan, R.; Leela, R.; Jubie, S.; Gowramma, B.; Gomathy, S.; Sankar, S. Ind. J. of Chem., 2011, 50(B), 1794-1799.

25. Liu M.; Sun, X.; Zhang, J. Arab. J. of Chem., 2017, 10(2), 167-171. 